\title{
Treatment strategies for left subclavian artery during total arch replacement combined with stented elephant trunk implantation
}

\author{
Zhenghua Xiao, MD, Wei Meng, MD, Da Zhu, MD, Yingqiang Guo, MD, and Eryong Zhang, MD
}

\begin{abstract}
Objective: One of the technical challenges in total arch replacement for type A aortic dissection is the left subclavian anastomosis and the descending aortic anastomosis. We present a technique that simplifies this surgery.

Methods: A total of 33 consecutive patients (mean age, $48.0 \pm 11.6$ years) with acute Stanford type A aortic dissection who underwent a total arch replacement were included in this study. Instead of anastomosing the graft to the descending aorta distal to the left subclavian artery, we ligated the left subclavian artery and performed the distal aortic anastomosis between the left carotid artery and the left subclavian artery. The left subclavian artery is then revascularized with a side arm from our aortic graft through the first intercostal space to the left axillary artery. The descending aorta also is stented in an antegrade fashion under direct visualization. Both intraoperative and postoperative complications were then noted.
\end{abstract}
Results: All enrolled patients underwent total arch replacement performed using this newly introduced tech- nique with a mortality rate of $18.2 \%(6 / 33)$. The causes of mortality were multiorgan failure (4 patients), renal failure ( 1 patient), and postoperative sepsis ( 1 patient). There was no intraoperative death and no anastomotic leak in our study cohort. Limb ischemia was observed in 4 patients, with all occurring in the lower limb. Postoperative delirium also was observed in 5 patients.

Conclusions: The left subclavian artery bypass technique during total arch replacement for type A dissection is reliable and simplifies the surgery by bringing the descending aortic anastomosis more proximal and eliminating the difficult left subclavian artery anastomosis. (J Thorac Cardiovasc Surg 2014;147:639-43)

Stanford type A aortic dissection is a lethal disease with an extremely poor prognosis, with a 2-week survival of less than $20 \%{ }^{1}$ Emergency type A aortic dissection is a challenging and complex surgery for cardiac surgeons because of the fragility of the tissues and instability of the patients, and it carries a high morbidity and mortality. ${ }^{2-4}$ Since introduced in 2002, total arch replacement combined with elephant trunk implantation and stenting of the descending aorta has became the standard treatment for type A aortic dissection. ${ }^{5,6}$ However, during total arch replacement, the left subclavian artery (LSA) anastomosis and the distal anastomosis at the descending aorta are often remarkably difficult because of their deep location in the surgical field. In addition, any anastomotic leak from these areas is difficult to visualize and address. ${ }^{7}$ Therefore, in this study, a treatment

\footnotetext{
From the Department of Cardiovascular surgery, West China Hospital, Sichuan University Chengdu, Sichuan, People's Republic of China.

Supported in part by Grant 81170288 from the National Research Foundation of Nature Science, China.

Disclosures: Authors have nothing to disclose with regard to commercial support. Drs Xiao and Meng contributed equally to this article.

Received for publication Aug 2, 2012; revisions received Sept 27, 2012; accepted for publication Feb 12, 2013; available ahead of print March 25, 2013.

Address for reprints: Eryong Zhang, MD, Department of Cardiovascular Surgery, West China Hospital, Chengdu, Sichuan, P. R. China 610041 (E-mail: zey16@ 126.com). $0022-5223 / \$ 36.00$

Crown Copyright (C) 2014 Published by Elsevier Inc. on behalf of The American Association for Thoracic Surgery

http://dx.doi.org/10.1016/j.jtcvs.2013.02.013
}

strategy is introduced for avoiding the LSA anastomosis and bringing the distal aortic anastomosis more proximal.

\section{MATERIALS AND METHODS \\ Patient Selection}

In this prospective cohort study, patients with Stanford type A dissection were enrolled in this study. Including criteria were (1) age 25 to 70 years and (2) acute type A dissection. Excluding criteria were (1) preoperative multiorgan failures that do not meet the criteria for surgical intervention, (2) age greater than 70 years, (3) dissection into the distal area of the LSA, (4) chronic aortic dissection, and (5) patient refusal to join the study. Informed consent was then obtained from each patient.

\section{Operative Method and Surgical Technique}

After induction of general anesthesia, arterial lines were inserted in bilateral radial arteries and femoral arteries. Operations were performed through a median sternotomy. The innominate artery, left common carotid artery (LCCA), and LSA were dissected and exposed step by step. Cardiopulmonary bypass was built through cannulation in the right axillary artery or left femoral artery and right atrium. Systemic cooling was then initiated The left ventricle was vented through the right superior pulmonary vein. The ascending aorta was then crossclamped, and the proximal part of the ascending aorta was opened. The heart was arrested with crystalloid cardioplegia directly down the coronary ostia, followed by intermittent blood cardioplegia every 30 minutes.

For repair of the ascending aorta, supracoronary aortic graft replacement, total ascending aortic replacement (Bentall or Carbol procedure), or aortic valve-sparing technique (annuloplasty) was performed according to the extent of the dissection and the status of the aortic valve during the cooling phase. When the nasopharyngeal temperature reached $22^{\circ} \mathrm{C}$ to $24^{\circ} \mathrm{C}$, the cardiopulmonary bypass was stopped. Then the innominate artery, LCCA, and LSA were clamped individually, and selective antegrade 


\section{Abbreviations and Acronyms \\ $\mathrm{CT}=$ computed tomography \\ LCCA $=$ left common carotid artery \\ LSA $=$ left subclavian artery}

cerebral perfusion was established through the right axillary artery and retrograde femoral arterial perfusion was discontinued. After inspection of the aortic arch, the aorta was transected between the LSA and the LCCA $^{9}$ (Figure 1). The stent graft (Shanghai MicroPort Medical Co, Ltd, Shanghai, China) (Figure 2, $A$ ) was then released antegrade into the descending aorta under direct visualization. The conventional straight aortic stent graft we used was $100 \mathrm{~mm}$ long and 26 to $36 \mathrm{~mm}$ in diameter, and there was a 10 -mm-long stent-free sewing margin. The diameter of stent graft selected was $10 \%$ to $20 \%$ larger than the size of the corresponding landing zone. End-to-end anastomosis was carried out between the distal end of the 4-branch vascular graft (Datascope Co, Montvale, NJ) (Figure 2, B) and the descending aorta. After anastomosis, the antegrade perfusion was reestablished through one of the side arms of the vascular graft.

After anastomosis of the left carotid artery to one of the side arms of the graft, the LSA was ligated in all cases because of its deep position. The patient was then rewarmed, and the crossclamp was removed. The heart started beating spontaneously or was otherwise stimulated by a defibrillator to recover normal sinus rhythm. Another 1 of the 4 side arm branches was anastomosed with the innominate artery and opened. Finally, the remaining side arm was passed through the first intercostal space into the left chest and anastomosed with the left axillary artery (Figure 3 ). Once the patient was fully rewarmed, cardiopulmonary bypass was weaned, hemostasis was controlled, and the chest was closed. The patient was then transferred to the intensive care unit.

\section{Outcome Measurement}

Perioperative mortality and complication, such as anastomosis leakage, organ malperfusion, limb ischemia, renal failure, and neurologic dysfunction, were all noted during hospital stay and follow-up. Enhanced chest computed tomography (CT) was performed for each patient before discharge, 3 months after surgical intervention, and once per year after discharge to evaluate the postoperative time course of the residual false lumen. All patients discharged from the hospital were followed up to the end of the study. The patients were contacted by telephone or direct interview in our outpatient clinic.

\section{RESULTS \\ Basic Data of Patients}

From February 2008 to December 2011, 33 patients with type A dissection treated through total arch replacement combined with stented elephant trunk implantation were enrolled in this study. Profiles of the patients are presented in Table 1. The average age was $48.0 \pm 11.6$ years (range, $22-59$ years), and $73 \%$ were male. All 33 patients had a diagnosis of acute type A aortic dissection, and surgery was performed within 14 days of clinical onset and within 4 days of hospital admission. The diagnosis was confirmed by $\mathrm{CT}$ and echocardiography. The most common risk factors are hypertension $(54.5 \%)$, smoking $(63.6 \%)$, and Marfan syndrome $(21.2 \%)$. The mean diameter of the ascending aortas was $56.6 \pm 0.6 \mathrm{~mm}$ (range, $43-73 \mathrm{~mm}$ ), and the mean left ventricular ejection fraction was
$47 \% \pm 9 \%$. The left ventricular end-diastolic diameter was 47 to $67 \mathrm{~mm}$, with a mean of $55.1 \pm 7.4 \mathrm{~mm}$. Moderate to severe aortic regurgitation also was observed in 18 cases. Two patients were intubated preoperatively for respiratory dysfunction, and 1 patient had impaired renal function. Intravenous antihypertensive drugs were given to all 33 patients to maintain a systolic pressure to within 100 to $120 \mathrm{~mm} \mathrm{Hg}$.

\section{Intraoperative Data}

Twenty patients required only ascending aortic replacement (in 5 patients, annuloplasty was performed to reconstruct the aortic valve), and 13 patients required the Bentall or Cabrol procedure. In 2 patients, coronary bypass grafting was carried out because the dissection involved the opening of the right coronary artery using a saphenous vein graft. The operative data are shown in Table 2. During surgery, the cardiopulmonary bypass time was $296.9 \pm 54.3$ minutes, aortic crossclamp time was $174.2 \pm 39.3$ minutes, deep hyperemia time was $52.2 \pm 33.2$ minutes, and mean nasopharyngeal temperature was $18.9^{\circ} \mathrm{C} \pm 2.7^{\circ} \mathrm{C}$ (range, $18.5^{\circ} \mathrm{C}-24.3^{\circ} \mathrm{C}$ ). The intraoperative data of the study cohort are shown in Table 2.

\section{Postoperative Data}

The in-hospital mortality rate was $18.2 \%$ (6/33). Four patients died of multiorgan failure caused by compromised visceral circulation as the result of malperfusion. The postoperative results are shown in Table 3. Ventilator time was $139.1 \pm 88.9$ hours (range, 62-230 hours). The patients remained in the intensive care unit for 3 to 25 days, with an average of $10.7 \pm 7.2$ days. The amount of chest tube drainage within 24 hours after surgery was 500 to $1240 \mathrm{~mL}$ with an average of $780 \pm 240.6 \mathrm{~mL}$. No paraplegia was noted among the study population. The total hospital stay was 16 to 52 days after surgery, with an average of $26.3 \pm 10.5$ days. Of the 33 patients, 4 died of multiple organ failure 11, 14, 18, and 26 days after surgery, 1 died of renal failure, and 1 died of postoperative sepsis. Six patients had pulmonary infection and left the hospital after treatment and recovery, and 5 patients had postoperative delirium but eventually resolved. The descending aortic wall in 20 patients visualized on postoperative CT scans had returned to normal with the residual false lumen gone at the time of discharge. Patent residual false lumen still existed in 7 patients at the region of the distal portion of the descending aorta and abdominal aorta.

\section{Follow-up}

Enhanced CT of the chest and abdomen was performed for each patient before discharge. All patients discharged from the hospital were followed up to the end of the study. The mean follow-up was $27.2 \pm 15.3$ months (range, 3-44 months). The long-term survival was $96.3 \%$ (26/27), and 


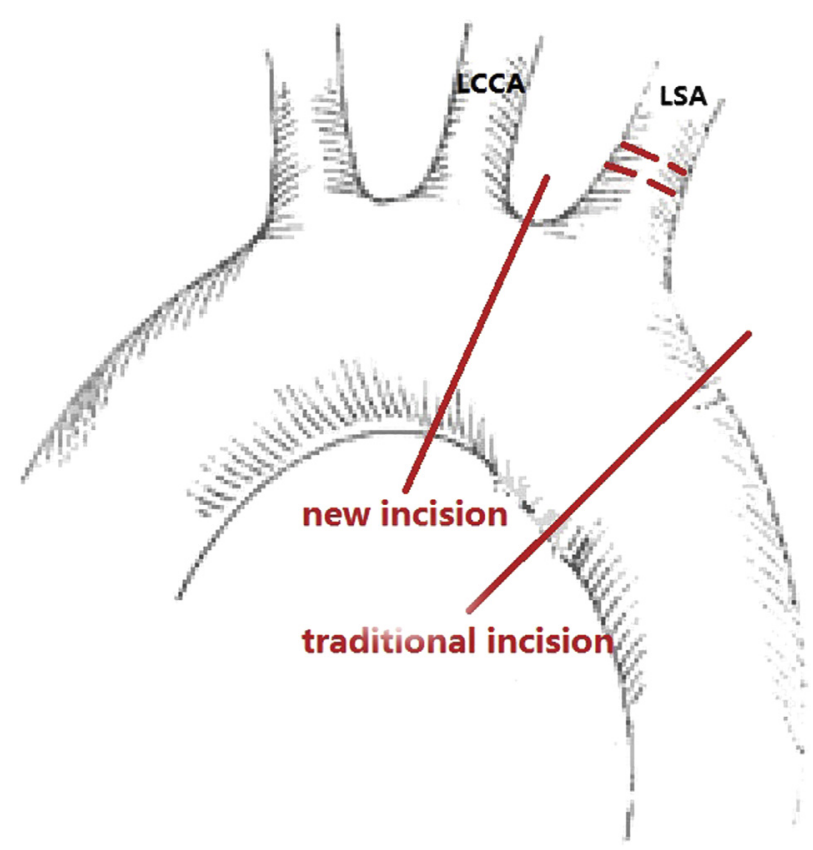

FIGURE 1. The proximal arch was transected between the LSA and the LCCA. The LSA was ligatured because of its deep position. LCCA, Left common carotid artery; LSA, left subclavian artery.

the freedom from reoperation rate was $92.6 \%$ (25/27). One patient died of progressive dilation of the false lumen of the aorta during aortic reoperation. One patient who had renal embolism as the result of thrombus from the false lumen died 10 months after the initial operation. The other patients resumed normal lives. Seventeen patients accepted antihypertensive therapy.

\section{DISCUSSION}

Total aortic arch replacement surgery is a complex surgery, especially in the setting of acute aortic dissection. Simplifying the surgery and minimizing bleeding are particularly important during aortic arch surgery. ${ }^{2-4}$ An original surgical technique for total arch replacement was formulated by several cardiovascular centers in recent years and showed encouraging outcomes. Kato and colleagues ${ }^{8}$ developed a classic hybrid technique in which the ascending aorta and aortic arch were replaced with a 4-branched graft, and a conventional straight stent graft was implanted into the descending aorta. However, the dissection often involves the orifice of the LSA, which was difficult to visualize because of its deep location in the surgical field. Then the distal anastomosis between the vascular graft and the descending aorta would be markedly difficult, especially in the fragile acute dissected aortic wall. ${ }^{7,9}$ The risk of anastomotic bleeding and repeat surgery for hemostasis still exists after the surgery even with careful manipulation and elaborate anastomoses during the procedure.

The LSA was directly ligatured in all 33 cases, and the distal aorta was transected circumferentially between the origin of the LCCA and the origin of the LSA. The distal aortic anastomosis was performed at the proximal arch between the LSA and the LCCA, which provided a better surgical view than the traditional anastomosis point in total arch replacement. The bleeding could be controlled easily if it occurred. Massive bleeding from the distal anastomosis site was not observed in any patients in our series. In addition, no patients required a reexploration of the chest to correct excessive postprocedural bleeding.

To simplify the surgery of aortic arch replacement, Chen and colleagues ${ }^{10}$ developed a single-stage hybrid procedure termed "open triple-branched stent graft placement technique for Stanford type A acute aortic dissection repair." However, a complication might still occur during deployment of the stent graft. Blind insertion of the stent graft posed the risk of creating a new intimal tear, and the stent graft could be twisted or kinked during the deployment, which could lead to an obvious endoleak. Shen and colleagues $^{11}$ also developed a triple-branched stent graft technique that made the surgical procedure easier, more time-saving, and less invasive; however, they also observed a stent graft endoleak during the application for acute aortic dissection. In our study, the stent graft is deployed antegrade under direct visualization, and postoperative CT scans showed that all stent grafts were fully opened with no kinking or twisting. Chen and colleagues ${ }^{12}$ used the
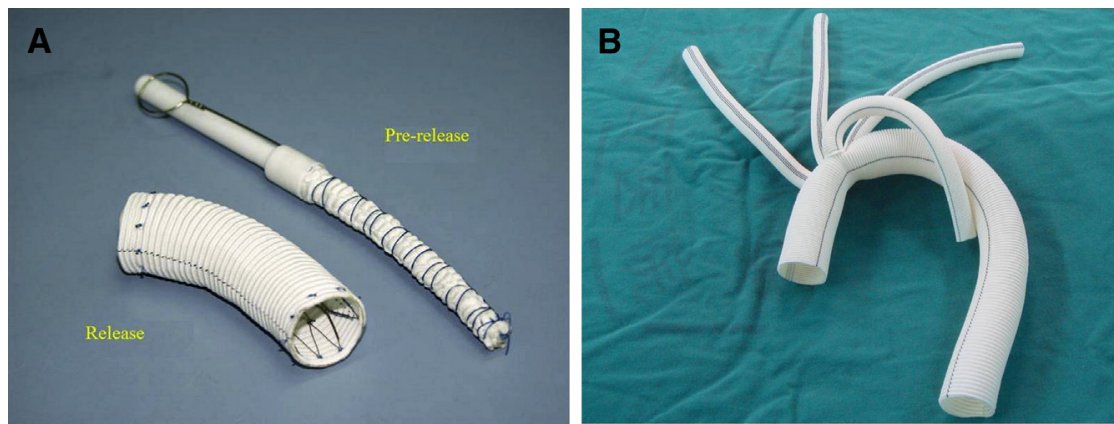

FIGURE 2. A, The stented graft (Shanghai MicroPort Medical Co, Ltd, Shanghai, China). B, The 4-branch artificial vessel (Datascope Co, Montvale, NJ). 

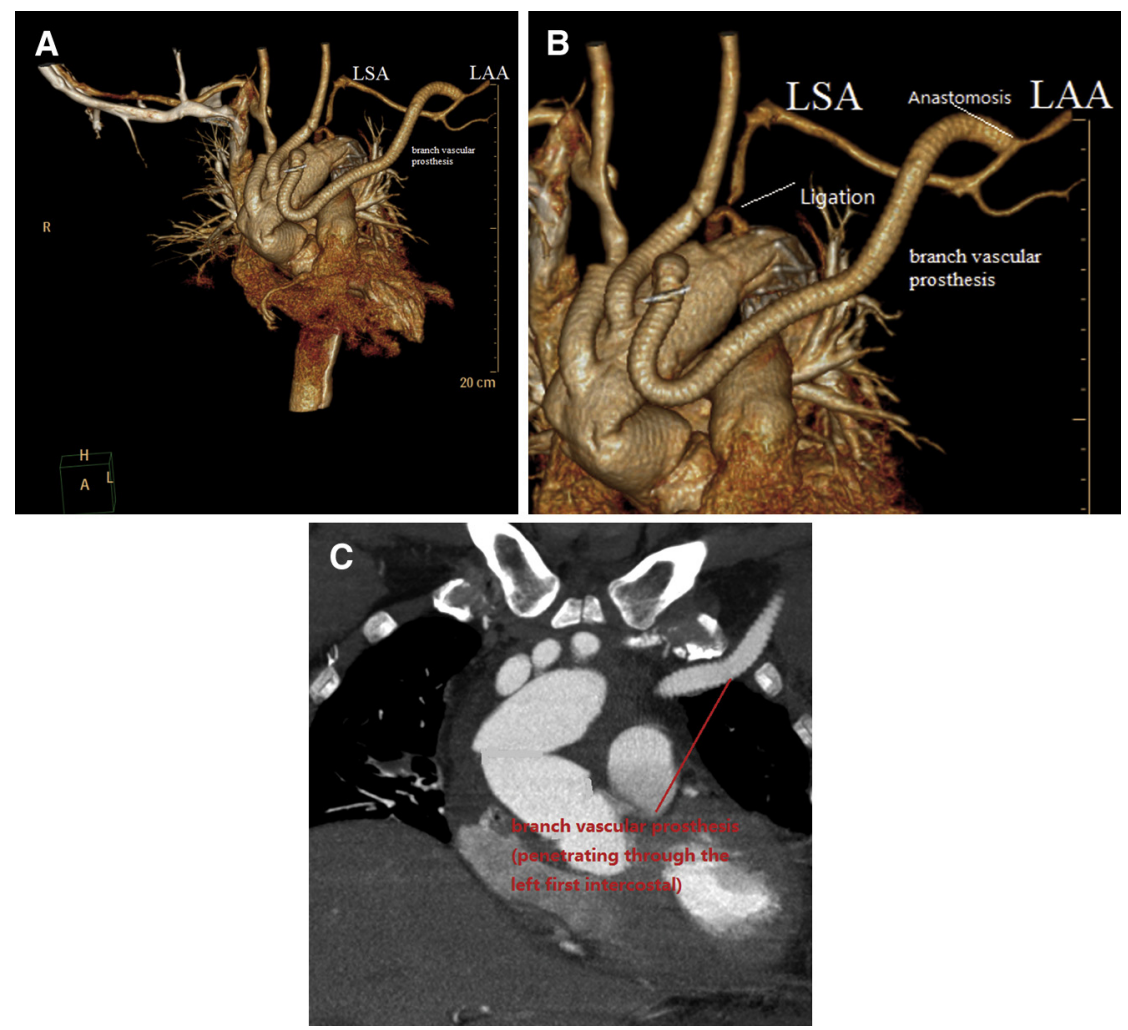

FIGURE 3. CT scans of the 3-dimensional reconstruction. A, Overview of the reconstructed ascending aorta, aortic arch, and descending aorta. B, Details of dealing with the LSA. C, CT scan shows branch vascular prosthesis penetrating through the left first intercostals. LSA, Left subclavian artery; LAA, left axillary artery.

single-branched stent-graft placement technique for avoiding the LSA anastomosis and the distal aortic anastomosis at the descending aorta, but this technique may not be suitable for the dissection caused by Marfan syndrome, because the future dilation of the LSA may lead to the malposition of stent graft. In our strategy, the transected distal stump of the aorta is stented from the inside and reinforced with Teflon felt on the outside, which eliminates the risk of malposition and endoleak formation in patients with Marfan syndrome.

TABLE 1. Basic data of patients

\begin{tabular}{lc}
\hline Sex (male/female) & $24 / 9$ \\
Age range (y) & $48.0 \pm 11.6$ \\
Mean \pm SD & \\
$\quad$ Marfan syndrome & $7(21.2 \%)$ \\
Hypertension & $18(54.5 \%)$ \\
Smoking & $21(63.6 \%)$ \\
Preoperative & \\
Left ventricular fractions (\%) & $47 \pm 9$ \\
Diameters of the ascending aortas (mm) & $56.6 \pm 0.6$ \\
Left ventricular end-diastolic diameters (mm) & $55.1 \pm 7.4$ \\
Aortic regurgitation & $18(54.5 \%)$ \\
Mechanical ventilation before operation & $2(6 \%)$ \\
Renal dysfunction & $1(3 \%)$ \\
\hline$S D$, Standard deviation.
\end{tabular}

During the follow-up, no such event was found in the 7 patients with Marfan syndrome.

Occurrence of recurrent nerve injury after arch surgery has been reported to be an independent predictor of postoperative pulmonary complications and may lead to longer duration of hospital stay. ${ }^{13}$ Tominaga and colleagues ${ }^{14}$ reported that the incidence of recurrent laryngeal nerve lesion was $9.1 \%$ in total arch replacement. No patient in our series had that specific complication. Postoperative vocal cord paralysis was not observed in our patients who underwent

TABLE 2. Operative profiles of patients

\begin{tabular}{lc}
\hline Operative procedure $(\mathrm{n})$ & \\
Aortic replacement & $15(45.5 \%)$ \\
Ascending & $5(15.2 \%)$ \\
Ascending + AVP & $4(12.1 \%)$ \\
Cabrol & $9(27.2 \%)$ \\
Bentall & $2(6 \%)$ \\
Coronary artery bypass grafting & $18.9 \pm 2.7$ \\
Lowest nasopharyngeal temperature $\left({ }^{\circ} \mathrm{C}\right)$ & $174.2 \pm 39.3$ \\
Aortic crossclamp time (min) & $296.9 \pm 54.3$ \\
CPB time (min) & $52.2 \pm 33.2$ \\
DHCA + SCP time (min) & \\
\hline$A V P$, Aortic valvuloplasty; $C P B$, cardiopulmonary bypass; $D H C A$, deep hypothermic \\
circulatory arrest; $S C P$, selective cerebral perfusion.
\end{tabular}


TABLE 3. Postoperative profiles of the patients

\begin{tabular}{lc}
\hline Postoperative profiles & \\
Respiratory support time (h) & $139.1 \pm 88.9$ \\
ICU stay time (d) & $10.7 \pm 7.2$ \\
Drainage within 24 h after surgery (mL) & $780 \pm 240.6$ \\
Complications & \\
Stroke or paraplegia (n) & 0 \\
Multiple organ failure & $4(12.1 \%)$ \\
Renal failure & $1(3 \%)$ \\
Sepsis & $1(3 \%)$ \\
Pulmonary infection & $6(18.2 \%)$ \\
Delay recovery & $5(15.2 \%)$ \\
Lower-limb ischemia & $4(12.1 \%)$ \\
\hline
\end{tabular}

$I C U$, Intensive care unit.

reconstruction of the LSA, mainly because the dissection, incision, and sutures of the distal arch and the descending aorta were no longer necessary as in this technique, and sequentially recurrent nerve injury was avoided. The branch vessel is anastomosed with the left axillary artery, which may reduce the risk of damaging the recurrent laryngeal nerve. By using this new procedure, we can avoid the LSA anastomosis and the distal anastomosis at the descending aorta.

Ligature of the LSA can lead to vascular and neurologic complications. The incidence of left-arm ischemia has been reported to be approximately $36 \% .{ }^{15}$ Symptoms are mostly transient and consist of a cooler hand, exercise-induced paresthesias of the left arm and hand, chest pain, or even distal digital trophic changes. In our study, the 1-branch of the 4-branch artificial vessel penetrated through the first intercostal and the left chest and then anastomosed with the side of the left axillary artery in 33 patients. This suggests that prophylactic LSA revascularization could effectively prevent left-arm ischemia. Lower-limb ischemia was noted in 4 patients, which may be due to the aortic dissection involving the femoral artery.

\section{Study Limitations}

This study is subject to the limitations inherent in clinical trials, that is, nonrandomized designs and relatively small sample sizes may affect the results because of the individual patient's consent and condition. This series is a report of a single institutional experience, and the results may not be generalized to other settings.

\section{CONCLUSIONS}

The LSA bypass technique during total arch replacement combined with stented elephant trunk implantation is a reliable technique that can simplify the procedure by bringing the distal aortic anastomosis more proximal and avoiding the difficult LSA anastomosis.

\section{References}

1. Coady MA, Rizzo JA, Goldstein LJ, Elefteriades JA. Natural history, pathogenesis, and etiology of thoracic aortic aneurysms and dissections. Cardiol Clin 1999;17:615-35.vii.

2. Fuster V, Ip JH. Medical, aspects of acute aortic dissection. Semin Thorac Cardiovasc Surg. 1991;3:219-24.

3. Hata M, Sezai A, Yoshitake I, Wakui S, Takasaka A, Minami K, et al. Clinical trends in optimal treatment strategy for type a acute aortic dissection. Ann Thorac Cardiovasc Surg. 2010;16:228-35.

4. Hagl C, Khaladj N, Peterss S, Bonz A, Pichlmaier M, Haverich A, et al. Treatment of acute aortic dissection type A (AADA): technical considerations. Vasa. 2010;39:212-8.

5. Chen X, Huang F, Xu M, Wang L, Jiang Y, Xiao L, et al. The stented elephan trunk procedure combined total arch replacement for Debakey I aortic dissection: operative result and follow-up. Interact Cardiovasc Thorac Surg. 2010; 11:594-8.

6. Dobrilovic N, Elefteriades JA. Stenting the descending aorta during repair of type A dissection: technology looking for an application? J Thorac Cardiovasc Surg. 2006;131:777-8.

7. Chen LW, Dai XF, Yang GF, Zhang GC, Cao H, Wang QM. Open-branched stent graft placement makes total arch replacement easier for acute type a aortic dissection. Ann Thorac Surg. 2010;89:1688-90.

8. Kato M, Kuratani T, Kaneko M, Kyo S, Ohnishi K. The results of total arch graft implantation with open stent graft placement for type A aortic dissection. J Thorac Cardiovasc Surg. 2002;124:531-40.

9. Mori Y, Hirose H, Takagi H, Umeda Y, Fukumoto Y, Shimabukuro K, et al Aortic arch repair for Stanford type A aortic dissection with distal anastomosis to the proximal level of the distal aortic arch. J Thorac Cardiovasc Surg. 2003;126:415-9.

10. Chen LW, Dai XF, Lu L, Zhang GC, Cao H. Extensive primary repair of the thoracic aorta in acute type A aortic dissection by means of ascending aorta replacement combined with open placement of triple-branched stent graft early results. Circulation. 2010;122:1373-8.

11. Shen K, Tang H, Jing R, Liu F, Zhou X. Application of triple-branched stent graft for Stanford type A aortic dissection: potential risks. Eur J Cardiothorac Surg. 2012;41:e12-7.

12. Chen LW, Dai XF, Yang GF, Zhang GC, Cao H, Wang QM. Open-branched stent graft placement makes total arch replacement easier for acute type A aortic dissection. Ann Thorac Surg. 2010;89:1688-90.

13. Ohta N, Kuratani T, Hagihira S, Kazumi K, Kaneko M, Mori T. Vocal cord paralysis after aortic surgery: predictors and clinical out come. J Vasc Surg. 2006;43: 721-8.

14. Tominaga R, Kurisu K, Ochiai Y, Nakashima A, Masuda M, Morita S, et al. Tota aortic arch replacement through the L-incision approach. Ann Thorac Surg. 2003; 75:121-5.

15. Weigang E, Parker JA, Czerny M, Lonn L, Bonser RS, Carrel TP, et al. Should intentional endovascular stent-graft coverage of the left subclavian artery be preceded by prophylactic revascularization? Eur J Cardiothorac Surg. 2011;40: 858-68. 